\title{
Fuzzy-logic assessment of failure hazard in pipelines due to mining activity
}

\author{
A. A. Malinowska and R. Hejmanowski \\ AGH University of Science and Technology, Cracow, Poland \\ Correspondence to: A. A. Malinowska (amalin@agh.edu.pl) \\ Published: 12 November 2015
}

\begin{abstract}
The present research is aimed at a critical analysis of a method presently used for evaluating failure hazard in linear objects in mining areas. A fuzzy model of failure hazard of a linear object was created on the basis of the experience gathered so far. The rules of Mamdani fuzzy model have been used in the analyses. Finally the scaled model was integrated with a Geographic Information System (GIS), which was used to evaluate failure hazard in a water pipeline in a mining area.
\end{abstract}

\section{Introduction}

Water pipelines localized in active mining areas are particularly hazarded with factors which may potentially evoke additional strains. Previous work shows that such factors include: seismic tremors, landslides, and continuous and discontinuous deformations of terrain due to underground extraction (Kalisz, 2007; Knothe, 1953; Kowalski and Kwiatek, 1995; Kwiatek and Mokrosz, 1996; Mendec et al., 1996; Mokrosz and Zawora, 1997; Szadziul, unpublished data; Talesnick and Baker, 2008; Zhao et al., 2005). Therefore, the failure-prone zones hazarding the pipelines, need to be managed because of the potential risk they bring. Depending on the significance of a given object, various evaluation methods are used for assessing the risk in particular situations. In the case of objects, whose failure may threaten the general safety and well-being of people, the applied methods are both time-consuming and very costly. Among the most frequent methods of the strain modeling is Finite Element Method (FEM; Zhao et al., 2005). Another approach is applicable when the linear objects are numerous and their failure may be only noxious for the users. In this case the applied estimation methods allow for a quick but approximated estimation of hazard. Unfortunately, approximated methods are inaccurate, and still time consuming. Therefore an attempt to work out a fuzzy model for assessing hazards caused by continuous deformations acting on linear objects was made. This model is integrated with GIS which significantly accelerates and simplifies the evaluation and reporting about the zones potentially threatened by underground extraction operations.

\section{Background}

\subsection{Pipeline hazard estimation - approximated point method}

In Poland, the strength of water pipelines in mining areas is evaluated with the point method. This method was created in the 1950s and has been commonly applied by the coal mine industry. This method offers an approximated evaluation of failure hazard of pipelines and consists of three stages:

1. resistance evaluation of water pipeline (expressed as resistance category (Table 1);

2. prediction of continuous strains hazard with Knothe function theory (expressed as terrain category) (Knothe, 1953);

3. comparison of terrain category with pipeline resistance category.

The resistance of the pipelines is assessed on the basis of four risk factors, and each of them is ascribed a certain number of points. On this basis the pipeline is given its category of resistance to horizontal strains. The higher is the number of points, the lower is the resistance category. A low resistance category means that a linear object is vulnerable and 
Table 1. Resistance classification of water pipelines - point method.

\begin{tabular}{|c|c|c|c|c|}
\hline \multicolumn{2}{|l|}{ Hazard factor } & \multicolumn{3}{|c|}{ Number of points } \\
\hline \multirow[t]{4}{*}{ Matter } & PE & & $0-10$ & \\
\hline & Cast iron or steel & & $10-15$ & \\
\hline & PCV & & $15-20$ & \\
\hline & Asbestos & & $20-30$ & \\
\hline \multirow[t]{3}{*}{ Compensation } & Pipe sleeve & & $0-10$ & \\
\hline & Compensators & & $10-20$ & \\
\hline & No compensators & & $20-30$ & \\
\hline \multirow[t]{4}{*}{ Type and number of connections } & $<1$ connection $100 \mathrm{~m}^{-1}$ & & $0-10$ & \\
\hline & $1-3$ connection $100 \mathrm{~m}^{-1}$ & & $10-20$ & \\
\hline & $3-5$ connection $100 \mathrm{~m}^{-1}$ & & $20-30$ & \\
\hline & $>5$ connection $100 \mathrm{~m}^{-1}$ & & $30-40$ & \\
\hline \multirow[t]{4}{*}{ Technical condition } & Very good & & $0-10$ & \\
\hline & Good & & $10-20$ & \\
\hline & Acceptable & & $20-30$ & \\
\hline & Poor & & $30-40$ & \\
\hline Number of points & $0-24$ & $25-48$ & $49-80$ & $>80$ \\
\hline Resistance category & 4 & 3 & 2 & 1 \\
\hline Acceptable horizontal strains $\left[\mathrm{mm} \mathrm{m}^{-1}\right]$ & 9.0 & 6.0 & 3.0 & 1.5 \\
\hline
\end{tabular}

prone to surface strains. In this case the probability of a failure occurrence is higher.

The terrain hazard is defined on the basis of predicted horizontal strains and tilts (Knothe, 1953). Then the terrain, where continuous deformations are possible, is categorized. Six categories have been distinguished depending on the intensity of predicted maximal horizontal strain $\varepsilon$ max:

1. $0\left(\varepsilon \max \in 0-0.3 \mathrm{~mm} \mathrm{~m}^{-1}\right)$;

2. I $\left(\varepsilon \max \in 0.3-1.5 \mathrm{~mm} \mathrm{~m}^{-1}\right)$;

3. II $\left(\varepsilon \max \in 1.5-3.0 \mathrm{~mm} \mathrm{~m}^{-1}\right)$;

4. III $\left(\varepsilon \max \in 3.0-6.0 \mathrm{~mm} \mathrm{~m}^{-1}\right)$;

5. IV $\left(\varepsilon \max \in 6.0-9.0 \mathrm{~mm} \mathrm{~m}^{-1}\right)$;

6. $\mathrm{V}\left(\varepsilon \max >9.0 \mathrm{~mm} \mathrm{~m}^{-1}\right)$.

The terrain categories are compared with pipeline resistance categories and on this basis the ultimate assessment is made.

\subsection{Estimation of continuous deformation hazard in linear objects - shortcomings}

The method used in Poland for evaluating hazard with continuous deformations in linear objects is very simplified. The assessment lies in finding objects where, the resistance category is exceeded by the terrain category (Fig. 1) (Szadziul, unpublished data).

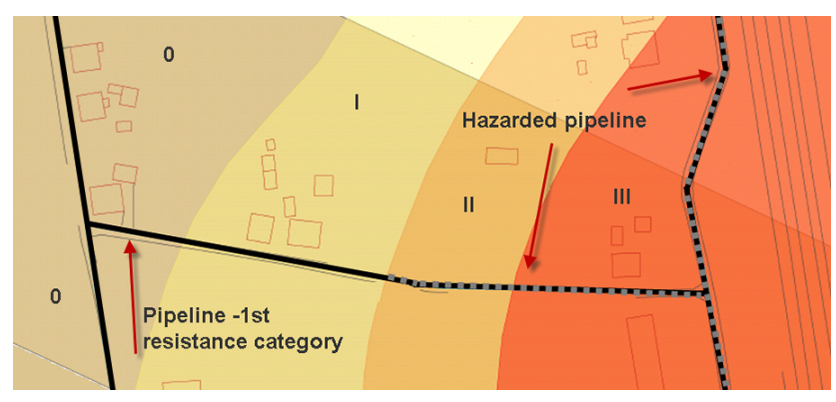

Figure 1. Example of the course of a linear object through terrains of belonging to different terrain categories $(0$, I, II, and III represent different terrain categories, with III being the most hazardous).

The practical application of this method has been exemplified below. A water pipeline belonging to the first resistance category is deposited in a ground of category 0-III (Fig. 1). Horizontal strains of terrain in that zone range between $0.1 \mathrm{~mm} \mathrm{~m}^{-1}$ (0 terrain category) to $4.5 \mathrm{~mm} \mathrm{~m}^{-1}$ (III terrain category). The pipeline can withstand horizontal strains under $1.5 \mathrm{~mm} \mathrm{~m}^{-1}$ (Table 1), therefore is hazarded in the terrain belonging to the II and III terrain category.

The major shortcoming of the presently applied method is discretisation of the described hazard. Moreover, the resistance of pipelines is defined in a very general way. Two sections of water pipeline ( $5 \mathrm{~m}$ from each other) can be evaluated as hazarded and not hazarded. Such discretisation creates serious problems to the organization responsible for safety in 


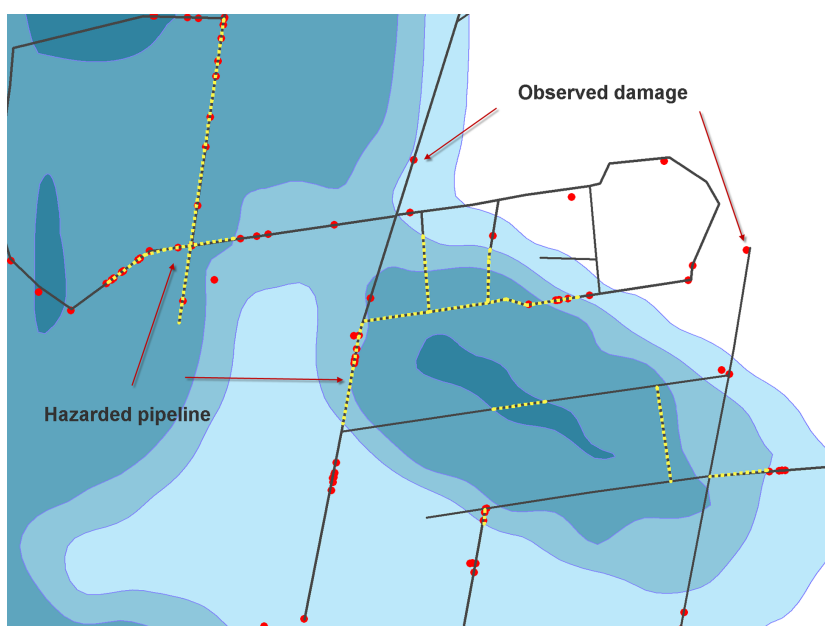

Figure 2. Hazard evaluation of water pipeline with point method (yellow dotted line: endangered part of the pipeline, red dot: observed damage).

mining areas, which have to make decisions about possible hazards and replacements of particular pipeline sections. As visualized in the presented example, the congruence between actual failures and places of potential hazard is low (Fig. 2).

Another problem lies in the lack of direct connection between terrain categories and pipeline resistance categories. Moreover, the present methodology is burdened with high uncertainty stemming from a number of factors including: incomplete information about the mining-geology conditions, incorrect modeling of continuous deformations, subjective evaluation of pipeline resistance; and, incomplete information about pipelines. This prompted the current study to identify other solutions, which would increase the efficiency of evaluation of pipelines hazard in areas staying under constant deformations of terrain.

\section{Modeling of pipeline hazard with fuzzy logic}

The analyses were focused on assuming additional risk factor for the assessment of pipeline hazard and developing a fuzzylogic model based on risk factors.

Fuzzy logic has been used in analyses since the 1960s (Zadeh, 1965). Initially it was used in industry for image processing, complex processes control, and computer-aided decision making. In the subsequent years attempts were undertaken to implement fuzzy theory in social systems, economy and even medicine. In environmental sciences fuzzy theories were used for evaluating hazards which could be generated by selected natural elements (Adriaenssensa et al., 2009; Bojorquez-Tapia et al., 2002; Busch and Maas, 2006; Gheorghe et al., 2000; Liu et al., 2005), e.g. for evaluating the landslide hazard (Lee, 2007), and evaluation of environmental elements in the aspect of their degradation (e.g., hazard evaluation of a river ecosystem, Ioannidou et al., 2003). It
Table 2. Rule base.

\begin{tabular}{|c|c|c|c|c|c|c|c|c|}
\hline & & \multicolumn{6}{|c|}{ HSM/HSR } \\
\hline & & & 0 & I & II & III & IV & $\mathrm{V}$ \\
\hline \multirow{5}{*}{ V } & & & $\mathrm{L}$ & VL & $\mathrm{M}$ & $\mathrm{H}$ & $\mathrm{VH}$ & $\mathrm{EH}$ \\
\hline & 4 & $\mathrm{~L}$ & $\mathrm{La}$ & $\mathrm{La}$ & $\mathrm{La}$ & VL & $\mathrm{L}$ & $\mathrm{M}$ \\
\hline & 3 & VL & $\mathrm{La}$ & $\mathrm{La}$ & VL & $\mathrm{L}$ & $\mathrm{M}$ & $\mathrm{A}$ \\
\hline & 2 & $\mathrm{M}$ & $\mathrm{La}$ & VL & $\mathrm{L}$ & $\mathrm{M}$ & A & $\mathrm{H}$ \\
\hline & 1 & $\mathrm{H}$ & VL & $\mathrm{L}$ & $\mathrm{M}$ & $\mathrm{A}$ & $\mathrm{H}$ & $\mathrm{VH}$ \\
\hline
\end{tabular}

should be emphasized that in a majority of cases the fuzzy analyses were supported by GIS tools. The present work represent the first time fuzzy logic has been used for assessing hazard assessment in pipelines placed in mining areas.

The first stage of creating a reasoning fuzzy system was determining input and output variables of the model. Basing on the studies by Malinowska et al. (unpublished data) two variables regarding the linear objects hazard were defined: predicted maximum horizontal strains $(\operatorname{HSM} \in(0,9))$ and the difference between predicted major strains in two directions $(\operatorname{HSR} \in(0,18)$. Another linguistic variable was the sensitivity of linear objects $(V)$, expressed as resistance points $(V \in(0,100)$. The failure hazard of a linear object expressed in the point scale $(R \in(0,100))$ was the output variable. This variable can be used for determining the failure hazard of a pipeline in points. The space in which these variables were analyzed was assumed on the basis of extreme values observed in the mining areas in Poland. Then the linguistic values were defined for each of the variables (Table 1): very low (VL), low (L), medium $(\mathrm{M})$, high $(\mathrm{H})$, very high $(\mathrm{VH})$, extremely high $(\mathrm{EH})$.

Pipeline vulnerability was described by 4 linguistic variables: very low $(\mathrm{VL})$, low $(\mathrm{L})$, medium $(\mathrm{M})$, high $(\mathrm{H})$, very high (VH).

Hazard of pipeline failure was characterized by 7 linguistic variables: lack (La), very low (VL), low (L), medium (M), appreciable (A), high $(\mathrm{H})$, very high $(\mathrm{VH})$.

Each linguistic value has a defined fuzzy set described by a characteristic function. The information which could describe the characteristic function is very limited, therefore authors assumed a triangle shape of the membership function for fuzzy sets. The membership functions were modeled based on the following assumptions:

1. characteristic points of the membership function are defined on the basis of limits of terrain category and resistance category,

2. membership functions meet the unity condition.

Then, a rule base was defined on the following assumptions (Table 2). 


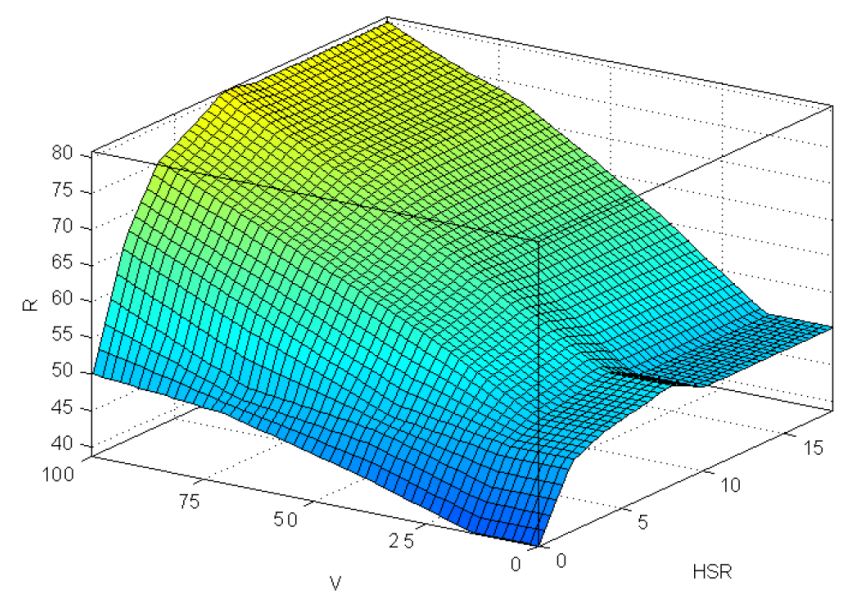

Figure 3. Surface of fuzzy model ( $V$ vs. HSR).

1. If the terrain category is higher than the resistance category by two or more grades, the failure hazard is serious.

2. If the terrain category is equal to the resistance category, the failure hazard is very low.

Fuzzy modelling was realized with the use of the Mandami dependence. The degree to which the premises in the inference block have been met was evaluated with the product operator (PROD). The successively activated premises allowed for determining the degree to which conclusions have been met. The last stage of fuzzy reasoning lied in determining the output function of rule base conclusions. The output membership function was defined on the basis of membership functions of particular conclusions of rules in the accumulation process. The accumulation in the model was performed for the SUM operator. The output membership function obtained in the course of inference was used for determining a sharp output value representing this set in the most reliable manner. Defuzzification was performed with the gravity center method. On this basis a surface was created, allowing for the evaluation of pipeline hazard with continuous strains (Fig. 3).

\section{Model application}

Modeling of pipeline failure hazard is supported by GIS programming. This is of special significance in predominantly urbanized areas, where the water network is extensive. It should be stressed that a sound evaluation of the failure hazard with GIS can be done only when reliable data about the resistance of the pipeline and the expected deformations which may occur in the study area are available (Fig. 4).

In the proposed solution based on a fuzzy model hazard in pipelines can be determined in a continuous manner, for each segment of the pipeline (the length to be defined arbitrarily). The pipeline can be ascribed a point value of failure

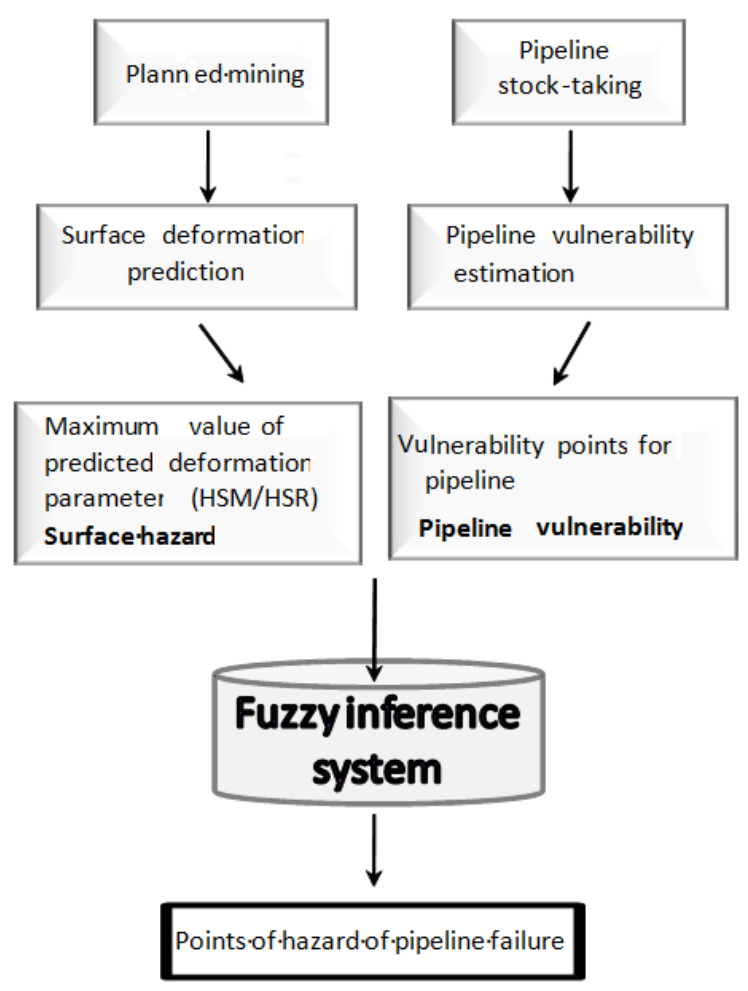

Figure 4. Pipeline damage risk assessment algorithm.

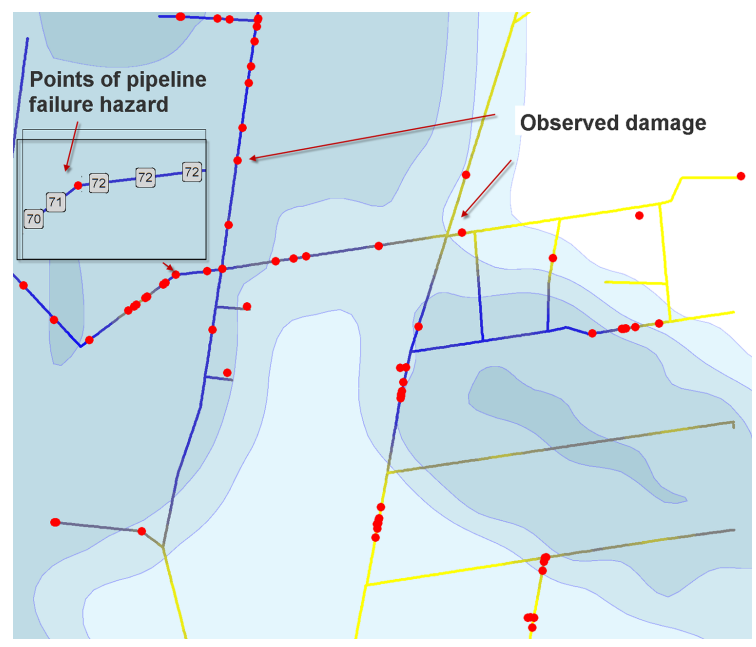

Figure 5. Evaluation of failure hazard of a pipeline performed with a fuzzy model (yellow dotted line: endangered part of the pipeline, red dot: observed damage, line with the blue intensive color-predicted high risk of pipeline damage).

risk $(R \in(0,100))$. The pipeline sections marked in intense colors are more endangered with failures (Fig. 5). The congruence between actual failures and places of potential hazard is high.

Organizations responsible for water pipelines are free to define the limits of risk points, for which the hazard is very 
high. The proposed model can be used for evaluating hazards in other linear objects, such as gas pipelines, when parameters have been adapted to the local conditions.

\section{Summary}

In a fuzzy-set based model the uncertainty resulting from the subjective evaluation of experts or incomplete data can be accounted for. The applied solution increases the efficiency of estimation of a water pipeline hazard. Moreover, thanks to the application of the inference block, the hazard can be presented as a continuous variable, which seems to be very advantageous compared to other methods. The comparison of modeling results with actual observations of damaged pipelines revealed a considerably higher accuracy using this new method. In addition, the presented method of a failure hazard evaluation for water pipelines seems to be readily and easily integrated with geographic information systems.

Acknowledgements. The research reported in this paper has been supported by a grant from the National Science Centre no. 2011/01/D/ST10/06958.

\section{References}

Adriaenssensa, V., Baetsb, B. D., Goethalsa, P. L. M., and Pauwa, N. D.: Fuzzy rule-based models for decision support in ecosystem management, Sci. Total Environ., 319, 1-12, 2009.

Bojorquez-Tapia, L. A., Juarez, L., and Cruz-Bello, G.: Integrating Fuzzy Logic, Optimization, and GIS for Ecological Impact Assessments, Environ. Manag., 30, 418-433, 2002.

Busch, W. and Maas, K.: Remarks to the risk assessment for abandoned mine sites, Acta Montanistica Slovaca Ročník 12, 3, 340348, 2006

Gheorghe, A. V., Mrock, R., and Kröger, W.: Risk assessment of regional systems, Reliab. Eng. Syst. Safe., 70, 141-156, 2000.

Ioannidou, I. A, Paraskevopoulos, S., and Tzionas, P.: Fuzzy modeling of Interactions among Environmental Stressors in the Ecosystem of Lake Koronia, Greece, Environ. Manag., 32, 624-638, 2003.

Kalisz, P.: The impact of the mining on the pipeline resiliency, Zeszyty Naukowe Politechnika Śląska S. Górnictwo, 278, 191200, 2007 (in Polish).

Knothe, S.: Equation of the profile of finitely formed subsidence trough, Archiwum Górnictwa i Hutnictwa, 1(1), 1953 (in Polish).

Kowalski, A. and Kwiatek, J.: Surface and surface structure object protection on the mining area, Przegląd Górniczy, 4, 11-19, 1995 (in Polish).

Kwiatek, J. and Mokrosz, R.: Gas networks on the mining areas, WUG, 3, 1996 (in Polish).

Lee, S.: Application and verification of fuzzy algebraic operators to landslide susceptibility mapping, Environ. Geol., 52, 615-623, 2007.

Liu, H., Chen, W., and Kang, Z.: Fuzzy Multiple Attribute Decision Making for Evaluating Aggregate Risk in Green Manufacturing, J. Tsinghua Sci. Technol., 10, 627-632, 2005.
Mendec, J., Kliszczewicz, B., and Wytrychowska, M.: Surface and surface structure object protection on the mining area, Rules for the water pipeline protection against the effects of underground mining, Glówny Instytut Górnictwa, Katowice, Poland, 53 pp., 1996 (in Polish).

Mokrosz, R. and Zawora, J.: The impact of the mining on the buried pipeline, Materiały konferencyjne: Ochrona powierzchni i o biektów budowlanych przed szkodami górniczymi, Glówny Instytut Górnictwa, Katowice, Poland, 20-28, 1997 (in Polish).

Talesnick, M. and Baker, R.: Failure of flexible pipe with a concrete liner, Eng. Fail. Anal., 5, 247-259, 2008.

Zhao, W., Nassar, R., and Hall, D.: Design and reliability of pipeline rehabilitation liners, Tunn. Undergr. Sp. Tech., 20, 203-212, 2005.

Zadeh, L. A.: Fuzzy sets, Inform. Control, 38, 1-14, 1965. 